UDC 576.311 .31

DOI: $10.20333 / 2500136-2021-2-104$

\title{
Targeting breast cancer exosomes with nucleic aptamers: innovative tools for early diagno- sis and therapy
}

C. L. Esposito ${ }^{1}$, C. Quintavalle ${ }^{1}$, F. Ingenito ${ }^{1}$, D. Rotoli ${ }^{1}$, G. Roscigno ${ }^{2}$, S. Nuzzo ${ }^{3}$, R. Thomas ${ }^{4}$, S. Catuogno ${ }^{1}$, Z. Minic ${ }^{5}$, M. Berezovski ${ }^{6}$, V. de Franciscis ${ }^{1}$, Gerolama Condorelli1, ${ }^{1,2}$

${ }^{1}$ Istituto di Endocrinologia ed Oncologia Sperimentale, Consiglio Nazionale delle Ricerche (CNR), Naples80100, Italy

${ }^{2}$ Department of Molecular Medicine and Medical Biotechnology, "Federico II" University of Naples, Naples 80100 , Italy

${ }^{3}$ IRCCS SDN, Naples 80100, Italy

${ }^{4}$ Mediterranea Cardiocentro, Naples 80100, Italy

${ }^{5}$ John L. Holmes Mass Spectrometry Facility, Faculty of Science, University of Ottawa, Ottawa ON K1N 6N5, Canada

${ }^{6}$ Department of Chemistry and Biomolecular Sciences, University of Ottawa, Ottawa ON K1N 6N5, Canada

Abstract. Exosomes are emerging as promising target for early diagnosis and therapy in different oncological conditions including breast cancer (BC). However, the development of tools able to easily and specifically target cancer cell-derived exosomes still represent a fundamental issue that is required to realize their clinical utility. Nucleic-acid aptamers are a promising class of structured single stranded oligonucleotides that serve as high affinity ligands of disease-associated proteins. Given their high potential in diagnosis and therapy, we addressed the development of aptamers specific for BC-derived exosomes. To this end, we developed a novel SELEX strategy by using exosomes purified from primary BC cells as positive selection target. By such a strategy we isolated nuclease resistant RNA aptamers able to specifically discriminate BC-derived exosomes from those produced by normal cells. The best sequences were optimized identifying short molecules (about 30-35 mer) that was characterized as tools for exosome detection. Further, we demonstrated that the developed aptamers inhibited exosome cellular uptake antagonizing cancer exosome-induced cell migration. By proteomic approach we identified possible targets that we are characterizing. Our results underline the great potential of isolated aptamers as tools for the development of innovative strategies for $\mathrm{BC}$ early diagnosis and therapy.

Key words: exosomes, aptamers, diagnosis, therapy, oncology.

Conflict of interest. The authors declare the absence of obvious and potential conflicts of interest associated with the publication of this article.

Citation: Esposito CL, Quintavalle C, Ingenito F, Rotoli D, Roscigno G, Nuzzo S, Thomas R, Catuogno S, Minic Z, Berezovski M, de Franciscis V, Condorelli Gerolama. Targeting breast cancer exosomes with nucleic aptamers: innovative tools for early diagnosis and therapy. Siberian Medical Review. 2021 ; (2):104. DOI: $10.20333 / 2500136-2021-2-104$

\section{Author information}

Carla L. Esposito, PhD, Istituto di Endocrinologia ed Oncologia Sperimentale, Consigli Nazionale delle Ricerche (CNR), 80100, Naples, Italy; e-mail: c.esposito@ieos.cnr. it Cristina Quintavalle, PhD, Istituto di Endocrinologia ed Oncologia Sperimentale, Consiglio Nazionale delle Ricerche (CNR), 80100, Naples, Italy; e-mail: cristina.quintavalle@ieos.cnr.it Francesco Ingenito, PhD, Istituto di Endocrinologia ed Oncologia Sperimentale, Consiglio Nazionale delle Ricerche (CNR), 80100, Naples, Italy. Deborah Rotoli, PhD, Istituto di Endocrinologia ed Oncologia Sperimentale, Consiglio Nazionale delle Ricerche (CNR), 80100, Naples, Italy. Giuseppina Roscigno, PhD, Department of Molecular Medicine and Medical Biotechnology,

"Federico II" University of Naples, 80100, Naples, Italy; e-mail: giusy_roscigno@yahoo.it Silvia Nuzzo, PhD, IRCCS SDN, 80100, Naples, Italy. Renato Thomas, Mediterranea Cardiocentro, 80100, Naples, Italy.

Silvia Catuogno, PhD, Istituto di Endocrinologia ed Oncologia Sperimentale, Consiglio

Nazionale delle Ricerche (CNR), 80100, Naples, Italy; e-mail: s.catuogno@ieos.cnr.it
Zoran Minic, PhD, John L. Holmes Mass Spectrometry Facility, Faculty of Science, University of Ottawa, Ottawa, ON K1N 6N5, Canada; e-mail: zminic@uottawa.ca

Maxim Berezovski, PhD, Department of Chemistry and Biomolecular Sciences, University of Ottawa, Ottawa, ON K1N 6N5, Canada; e-mail: maxim.berezovski@uottawa.ca

Vittorio de Franciscis, PhD, Istituto di Endocrinologia ed Oncologia Sperimentale, Consiglio Nazionale delle Ricerche (CNR), 80100, Naples, Italy; e-mail: defranci@unina.it

Gerolama Condorelli, PhD, Istituto di Endocrinologia ed Oncologia Sperimentale Consiglio Nazionale delle Ricerche (CNR), 80100, Naples, Italy; Department of Molecular Medicine and Medical Biotechnology, "Federico II" University of Naples, 80100, Naples, Italy; e-mail: gecondor@unina.it

Received 18 February 2021 Revision Received 18 March 2021 Accepted 31 March 2021 